\title{
DARBOUX'S THEOREM FAILS FOR WEAK SYMPLECTIC FORMS
}

\author{
J. MARSDEN ${ }^{1}$
}

Abstract. An example of a weak symplectic form on a Hilbert space for which Darboux's theorem fails is given.

Introduction. Let $\boldsymbol{E}$ be a Banach space and $B: E \times E \rightarrow R$ a continuous bilinear form. Let $B^{b}: E \rightarrow E^{*}$ be defined by $B^{b}(e) \cdot f=B(e, f)$. Call $B$ nondegenerate if $B^{b}$ is an isomorphism and call $B$ weakly nondegenerate if $B^{b}$ is injective. For a symmetric bilinear form $G$ on $\boldsymbol{E}$, define the skew form $\tilde{G}$ on $\boldsymbol{E} \times \boldsymbol{E}$ by

$$
\tilde{G}\left(\left(e_{1}, e_{2}\right),\left(f_{1}, f_{2}\right)\right)=G\left(f_{2}, e_{1}\right)-G\left(e_{2}, f_{1}\right) .
$$

It is easily seen that $\tilde{G}$ is nondegenerate (resp. weakly nondegenerate) iff $G$ is.

Now let $M$ be a Banach manifold. A symplectic form (resp. weak symplectic form) on $M$ is a smooth closed two form $\omega$ on $M$ such that for each $p \in M, \omega$ as a bilinear form on $T_{p} M$ is nondegenerate (resp. weakly nondegenerate); here $T_{p} M$ is the tangent space at $p$. Using a technique of Moser, Weinstein ([6], [7]) showed that for each $p \in M$ there is a local chart about $p$ on which $\omega$ is constant. This is a significant generalization and simplification of the classical theorem of Darboux. However, in many physical examples (the wave equation and fluid mechanics for instance) one deals with weak symplectic forms (see [1], [3], [4], [5]).

It is therefore interesting to know if Darboux's theorem remains valid for weak symplectic forms. In this note we give a counterexample.

Sympletic forms induced by metrics. If $M$ is a manifold, its cotangent bundle $T^{*} M$ carries a canonical symplectic form $\omega$. If $M$ is modeled on a reflexive space the form is nondegenerate; otherwise it is only weakly nondegenerate. See [1], [4]. Now let $\langle,\rangle_{p}$ be a (smooth) weak riemannian metric on $M$. Then it induces a map of $T M$ to $T^{*} M$. The pull back $\Omega$ of $\omega$ to $T M$ is called the form induced by the metric. It is a weak symplectic

Received by the editors June 4, 1971.

AMS 1970 subject classifications. Primary 58B20, 58F05.

Key words and phrases. Darboux's theorem, symplectic form.

${ }^{1}$ Partially supported by the National Research Council of Canada.

(c) American Mathematical Society 1972 
form and in a chart $U$ for $M$ it is given by (using principal parts):

$$
\begin{aligned}
2 \Omega_{u, e}\left(\left(e_{1}, e_{2}\right)\right. & \left.,\left(e_{3}, e_{4}\right)\right) \\
& =D_{u}\left\langle e, e_{1}\right\rangle_{u} \cdot e_{3}-D_{u}\left\langle e, e_{3}\right\rangle_{u} \cdot e_{1}+\left\langle e_{4}, e_{1}\right\rangle_{u}-\left\langle e_{2}, e_{3}\right\rangle_{u} .
\end{aligned}
$$

Here, $D_{u}$ denotes the derivative of the map $u \mapsto\left\langle e, e_{1}\right\rangle_{u}$ with respect to $u$. In the finite dimensional case this corresponds to the classical formula

$$
\Omega=\sum g_{i j} d q^{i} \wedge d \dot{q}^{j}+\sum \frac{\partial g_{i j}}{\partial q^{k}} \dot{q}^{i} d q^{j} \wedge d q^{k}
$$

Observe that in the finite dimensional case if we take new variables $q^{1}, \cdots, q^{n}, p_{1}, \cdots, p_{n}$ where $p_{i}=\sum g_{i j} \dot{q}^{j}$, then (as is easy to check) $\Omega=\sum d q^{i} \wedge d p_{i}$ which gives a chart in which $\Omega$ is constant.

The example. The following is a simplification of an earlier example. We thank the referee and Paul Chernoff for suggestions in this regard.

Let $\boldsymbol{H}$ be a real Hilbert space. Let $S: \boldsymbol{H} \rightarrow \boldsymbol{H}$ be a compact operator with range a dense, but proper subset of $\boldsymbol{H}$, which is selfadjoint and positive: $\langle S x, x\rangle>0$ for $0 \neq x \in \boldsymbol{H}$. For example if $\boldsymbol{H}=L_{2}(\boldsymbol{R})$, we can let $S=(1-\Delta)^{-1}$ where $\Delta$ is the Laplacian; the range of $S$ is $H^{2}(R)$.

Since $S$ is positive, -1 is clearly not an eigenvalue. Thus, by the Fredholm alternative, $a I+S$ is onto for any real scalar $a>0$. Define on $\boldsymbol{H}$ the weak metric $g(x)(e, f)=\left\langle A_{x} e, f\right\rangle$ where $A_{x}=S+\|x\|^{2} I$. Clearly $g$ is smooth in $x$, and is an inner product. Let $\Omega$ be the weak symplectic form on $\boldsymbol{H} \times \boldsymbol{H}=\boldsymbol{H}_{\mathbf{1}}$ induced by $g$, as was discussed above.

Proposition. There is no coordinate chart about $(0,0) \in \boldsymbol{H}_{1}$ on which $\Omega$ is constant.

Proof. If there were such a chart, say $\phi: U \rightarrow \boldsymbol{H} \times \boldsymbol{H}$ where $U$ is a neighborhood of $(0,0)$, then in particular in this chart, the range $F$ of $\Omega^{b}$, as a map of $\boldsymbol{H}_{1}$ to $\boldsymbol{H}_{1}^{*}$, would be constant. Let $B_{x, y}$ be the derivative of $\phi$ at $(x, y) \in \boldsymbol{H}_{1}$. Then we obtain that the range of $\Omega_{x, y}^{b}$ equals $B_{x, y}^{*} F$.

Now by the above formula for $\Omega$, at the point $(x, 0)$ we have

$$
2 \Omega_{(x, 0)}\left(\left(e_{1}, e_{2}\right),\left(e_{3}, e_{4}\right)\right)=g_{x}\left(e_{4}, e_{1}\right)-g_{x}\left(e_{2}, e_{3}\right) .
$$

But by construction, for $x \neq 0, g_{x}$ is a strong metric (i.e., $A_{x}$ is onto for $x \neq 0)$, so the range of $\Omega_{(x, 0)}^{b}$ is all of $H_{1}^{*}$ for $x \neq 0$. Since $B_{x, y}$ is an isomorphism, this implies that $\Omega_{(0,0)}^{b}$ is onto all of $\boldsymbol{H}_{1}^{*}$ as well. But $g_{0}$ is only a weak metric which is not onto as a map of $\boldsymbol{H}_{1}$ to $\boldsymbol{H}_{1}^{*}$. Hence $\Omega_{(0,0)}^{b}$ cannot be onto as well, a contradiction.

As was pointed out by the referee, the example even shows that $\Omega$ cannot be made constant on a continuous vector bundle chart on $T^{2} M \rightarrow$ $T M$, let alone by a manifold chart on $T M$. 
Of course the essense of the example is that the range of $\Omega$ suddenly changed at one point i.e., the topology of the metric suddenly changed. This is perfectly compatible with the smoothness of $\Omega$ as it is only a weak symplectic form. This suggests a possible conjecture pointed out by Paul Chernoff: If $\Omega$ is such that the ranges of $\Omega_{u}$ are locally equivalent via an isomorphism, then Darboux's theorem should hold. This can be verified directly in case $\Omega$ comes from a metric which has locally equivalent ranges.

\section{REFERENCES}

1. P. Chernoff and J. Marsden, Hamiltonian systems and quantum mechanics (in preparation).

2. J. M. Cook, Complex Hilbertian structures on stable linear dynamical systems, J. Math. Mech. 16 (1966), 339-349. MR 34 \#625.

3. D. Ebin and J. Marsden, Groups of diffeomorphisms and the motion of an incompressible fluid, Ann. of Math. (2) 92 (1970), 102-163.

4. J. Marsden, Hamiltonian one parameter groups, Arch. Rational Mech. Anal. 28 (1967/68), 362-396. MR 37 \#1735.

5. I. Segal, Conjugacy to unitary groups within the infinite dimensional symplectic group, Technical Report, Argonne National Laboratory, Argonne, Illinois, 1966.

6. A. Weinstein, Symplectic manifolds and their Lagrangian submanifolds, Advances in Math. 6 (1971), 329-346.

7. _ Symplectic structures on Banach manifolds, Bull. Amer. Math. Soc. 75 (1969), 1040-1041.

Department of Mathematics, University of Toronto, Toronto, Ontario, CANADA

Current address: Department of Mathematics, University of California, Berkeley, California 94720 